\title{
Revisión por pares en la Revista Médica de Chile, año 2011
}

\author{
Peer-reviewers in Revista Médica de Chile, year 2011
}

\begin{abstract}
T os revisores externos de los manuscritos que se reciben en la Revista Médica de Chile cumplen una labor voluntaria, desinteresada y anónima (para los autores). La "revisión por pares" es uno de los pilares que sostienen la calidad de las revistas científicas y se ha hecho cada día más importante al aumentar la complejidad metodológica de los trabajos, en el área científica y en las especialidades clínicas. Esta colaboración demanda tiempo, esfuerzo, la revisión de bases de datos y de otras publicaciones, y la aplicación de un juicio valorativo, todo lo cual se sintetiza en un informe escrito que se pone a disposición de los editores. Es un trabajo que debe ser reconocido y valorado por los lectores de la Revista y por las instituciones en que trabajan dichos revisores. Por ese motivo, una vez al año y en el número de julio de la Revista, se identifican las personas que durante el año precedente revisaron al menos un manuscrito. Durante 2011 contamos con la participación de 587 revisores externos, la mayoría de los cuales revisaron dos o tres manuscritos en el
\end{abstract}

año. Quienes figuran en la nómina de Miembros del Comité Editorial Asesor de la Revista, revisaron generalmente un número aún mayor.

Los informes de los revisores externos prestan una ayuda inapreciable a los autores de manuscritos, cuando expresan críticas y sugerencias que les permiten corregir omisiones o errores y, por ende, mejoran la calidad de sus manuscritos. Los editores, a su vez, se apoyan en las opiniones de los revisores externos para decidir si aceptan o rechazan un manuscrito, o solicitan a los autores que hagan cambios antes de tomar su decisión final. Todo este proceso incide directamente en la calidad de la Revista, según la aprecien sus lectores $\mathrm{y}$ otros autores que aborden un mismo tema.

En nombre de los editores de la Revista Médica de Chile y del Directorio de la Sociedad Médica de Santiago-Sociedad Chilena de Medicina Interna, agradecemos a quienes revisaron manuscritos durante el año 2011. La nómina que se publica a continuación es un documento que acredita su participación en el proceso de "revisión por pares".

Listado revisores 2011

Fernando Abarzúa C

Alexis Aceituno A

Juan Carlos Acevedo B

Mónica Acevedo B

Patricia Acuña J

Guillermo Acuña L

Raúl Acuña M

Mónica Acuña P

Alejandro Afani S

Rubén Aguayo N

Andrés Aizman $S$

Ana María Alarcón M

Cecilia Albala B

Jorge Alfaro L

Ricardo Allegri

Felipe Aller R

\author{
María Angélica Alliende R \\ Faustino Alonso T \\ Rubén Alvarado M \\ Francisco Alvarez G \\ Gonzalo Alvarez U \\ Manuel Alvarez Z \\ Miriam Alvo A \\ Roberto Amador C \\ Hugo Amigo C \\ Miguel Antúnez R \\ Marcela Aracena A \\ Daniel Araos H \\ Teresa Aravena C \\ Pablo Aravena C \\ Fernando Araya D \\ Leonardo Arellano H
}

\author{
Marco Arrese J \\ Oscar Arteaga $\mathrm{H}$ \\ Antonio Arteaga Ll \\ Eugenio Arteaga U \\ Sylvia Asenjo M \\ Marcela Assef C \\ Eduardo Atalah S \\ Arturo Ayala B \\ Francisco Ayala R \\ Carmen Gloria Aylwin H \\ Hernán Baeza R \\ Claudia Bambs S \\ Guillermo Bannura C \\ Paulina Barría P \\ Manuel Barría S \\ Manuel Barros M
}


María de la Luz Bascuñán R Gabriel Bastías S

Rene Baudrand B

Paula Bedregal G

Fernando Bedregal del R

Rosa Behar A

Ernesto Behnke G

Sergio Bello S

Carlos Benítez G

Zoltan Berger $\mathrm{F}$

Pablo Bertin C

Mauricio Besio R

Leandro Biagini A

Marcelo Bianchi P

María Antonieta Blanco R

Justo Bogado S

Gisella Borzone T

Arturo Borzuztky S

María Lina Boza C

Javier Brahm B

Sergio Brantes G

Pablo Brockmann V

Lucía Bronfman F

Francisca Browne L

Alejandro Bruhn C

Daniel Bunout B

Paula Burgos C

Jaime Burrows $\mathrm{O}$

Raquel Burrows A

Eduardo Bustos O

Felipe Cabello C

Raúl Cabrera M

María Elena Cabrera C

Texia Cáceres C

Mario Calvo A

Jamile Camacho N

Mario Campero S

Mauricio Camus A

Mauricio Canals L

Carlos Caorsi S

María Cecilia Capetillo F

Jorge Carabantes C

Daniel Cárcamo P

Juan Guillermo Cárcamo M

Laura Carreño T

Luis Cartier R

Jorge Carvajal C

Sergio Carvajal C

Carlos Carvajal H

Pilar Carvallo de S-Q

Aurelio Carvallo V
Juan Casar L

Fernando Cassorla G

Gisella Castiglione V

Carolina Castillo $\mathrm{H}$

Manuel Castillo N

Octavio Castillo C

Carlos Castillo D

Cecilia Castillo L

Marianela Castillo R

Pablo Castro G

Gabriel Cavada Ch

Felipe Cavagnaro S

Silvana Cavallieri B

María Elena Ceballos V

Carlos Celedón L

Tulio Chacin P

Hernán Chamorro B

Gastón Chamorro S

Pedro Chaná C

Jorge Chávez A

Sara Chernilo St

José Chianale B

May Chomalí G

Mirtha Cifuentes M

Lucía Cifuentes O

Luis Ciocca G

José Miguel Clavero R

Miguelángel Clerc C

Ethel Codner D

Verner Codoceo R

Guillermo Conte L

Adela Contreras R

Jorge Contreras B

Héctor Contreras M

Jaime Contreras $\mathrm{P}$

Andrea Contreras S

Ramón Corbalán H

Claudia Cortés M

Esteban Cortés S

Alejandro Corvalán R

Javier Crosby R

Carlos Cruz M

Francisco Cruz O

Ada Cuevas M

Eduardo Cunill C

Karin D'Ottone M

Jeannette Dabanch P

Xabier De Aretxabala U

Flora De la Barra M

María Pía De la Maza C

Guillermo De la Parra C
Raúl De la Sotta T

Carla Delporte V

Rodrigo Díaz G

Carlos Díaz C

Jaime Díaz C

Julio Dölz V

Mariana Domínguez Y

Mario Donoso S

Patricio Downey C

Juan Dubernet M

Hector Ducci B

Mauricio Duhalde N

Pilar Durruty A

Ana Dvoredsky B

Anneliese Dörr A

Carlos Echeverría B

Ana Escribar W

Manuel Espinoza S

Ricardo Espinoza A

Ricardo Espinoza G

Mauricio Espinoza R

Ivo Eterovic M

Arturo Evangelista M

Alejandro Fajuri N

Patricia Fardella B

Rossana Faúndez $\mathrm{H}$

Manuel Fernández A

Wanda Fernández M

Alejandra Fernández V

Alberto Fica C

Juan Alberto Fierro C

Hernán Figueroa $\mathrm{V}$

Gustavo Figueroa C

Fernando Florenzano U

Ramón Florenzano U

Fernando Fluxá G

María Luisa Fonck L

Carmen Franco S

Ariel Fuentes G

Patricio Fuentes $G$

Homero Gac E

Marcia Gaete C

Verónica Gaete $\mathrm{P}$

Jorge Gajardo N

Sergio Gálvez G

Pilar Gambra A

Bernardita Garayar P

Flavia Garbin A

Patricia García C

Carlos García C

Ricardo García S 
María Luisa Garmendia M Héctor Gatica R Ricardo Gazitúa H Roger Gejman E Alfredo Germain A Juan Giaconi G Rodrigo Gil D José Giordano B Gonzalo Godoy J Alejandro Goic G Alejandro Gómez Ch Fernando Gómez L Ignacio Goñi E Julia González M Rolando González A Sergio González B Jorge González C Fernando González F Jorge González H Mauricio Gonzalez L Gerardo González R Gilberto González V Karen Goset P Marcos Goycoolea V Carlos Grant del R Douglas Greig U Monica Grez A Humberto Guajardo S Julia Guerrero P Rafael Guerrero P Eliana Guic S Ernesto Guiraldes C Miguel Gutiérrez T Christel Hanne A

Jorge Hasbun $\mathrm{H}$ Carmen Henríquez O María Isabel Herane $\mathrm{H}$ Carlos Hermansen T Denisse Hernández F Guillermo Hernández G Antonio Hernández M Rodrigo Hernández V Viviana Herskovic M Pedro Herskovic L Beatrice Hervé E Daniel Hinzpeter K Sandra Hirsch B María Isabel Hodgson B Ricardo Hojas B Juan Honeyman M Arnold Hoppe W
Nina Horwitz C Trinidad Hoyl M Álvaro Ibarra V Gloria Icaza N Juan Idiáquez C Juan Ilzauspe Z Antonio Infante B Franco Innocenti $\mathrm{C}$ Carla Inzunza C

Lorena Isbej E

Fernando Ivanovic-Zuvic R Enrique Jadresic M Liliana Jadue $\mathrm{H}$ Roberto Jalil M Alejandra Jara G Ivonne Jelves $\mathrm{M}$ Patricio Jiménez $\mathrm{P}$

María Isabel Jirón $\mathrm{V}$

Rodrigo Julio A

Carlos Juri $\mathrm{Cl}$

Karin Jürgens $S$ Mariana Kahn Ch Elena Kakarieka W

Francisco Kamann C Christian Karmelic S Julieta Klaassen L Owen Korn B Mariane Krause J Manuel Krauskopf R Daniela Krämer H

Gisela Kuester F Sonia Kunstmann F Juan Labra J

Rodolfo Lahsen M de la P Alexis Lama T

Fernando Lanas Z Martín Larico G Marcela Larraguibel Q Milton Larrondo L Jorge Lastra T Ricardo Latorre M Manuel Lavados M Richard Lee Isabel Leiva $\mathrm{R}$ Guillermo Lema F Augusto León R Lorena León B Luz María Letelier S Cristian Leyton M Federico Liendo P Patricia Lillo Z
Marcelo Llancaqueo V

Carolina Llanos M

Osvaldo Llanos L

Pedro Llorens S

Nelson Lobos J-F

Fernando Lolas St

Francesca Lombardo de T

Ilse López B

Francisco López K

Marcelo López L

Gloria López S

Néstor López V

Jaime Lubascher C

Ricardo Madariaga $\mathrm{R}$

Jorge Madrid A

Ana Maida $S$

Alberto Maiz G

Alejandro Majlis L

Marcelo Marconi T

Francisco Mardones S

Paula Margozzini M

María José Martínez G.

Virginia Martínez C

Claudio Martínez G

Benjamín Martínez R

Alejandro Martínez S

Víctor Martínez Z

Pablo Matamala B

Patricia Matus C

Marinella Mazzei P

Peter Mc Coll C

Raquel Medina K

Sergio Mella M

Cecilia Mellado S

Rómulo Melo $\mathrm{M}$

Francisco Mena C

Francisco Mena G

Juan Méndez V

Rubén Mercado P

Tomás Mesa L

Diego Mezzano A

Sergio Mezzano A

Luis Michea A

Teresa Millán K

Marcelo Miranda C

Harold Mix A

Montserrat Molgó N

Ramiro Molina C

Alfredo Molina P

Rodrigo Montagna $\mathrm{M}$

Hernán Montenegro A 
Edson Montero C Joaquín Montero L Adela Montero V

Philippa Moore Francisco Moraga M Miguel Morales S Rodrigo Moreno B Manuel Moreno G Raimundo Morris C Leonor Moyano S Patricia Muñoz C del V Gabriela Muñóz G Sergio Muñoz N Santiago Muzzo B Bettina Müller Sch Julio Nazer H Carolina Nazzal N

Oscar Neira Q Jorge Nogales G Luis Miguel Noriega $R$ Rebeca Northland A Fernando Novoa S David Oddó B Danny Oksenberg R Verónica Olavarría L Luis Oliva P Manuel Olivares G Cristina Olivos O Alfonso Olmos C Pablo Olmos C Luis Orlandi J Lina Ortiz L Manuel Oyarzún G Rodulfo Oyarzún F Miguel Oyonarte G Daniel Pacheco R Cristian Palma B Irma Palma M Karin Papapietro V María Teresa Parada C Enrique Paris M Claudio Parra R Walter Passalacqua R Alfredo Pemjean G Carlos Peña F Jaime Pereira G Patricia Pérez A Carlos Pérez C María Luisa Pérez Cotapos S Gilberto Pérez P Manuel Pérez-Flores
Fernando Pimentel M

Gracia Pinheiro de C

Paulina Pino Z

María Eugenia Pinto $\mathrm{Cl}$

Ricardo Pinto M

Luis Plubins M

Jose Poblete L

Felipe Pollak C

Lorena Porte T

Juan Carlos Prieto D

Bárbara Puga L

Klaus Püschel I

Rodrigo Quera P

Iván Quevedo L

Arnoldo Quezada L

Juan Carlos Quintana F

Ricardo Rabagliati B

Gabriel Rada G

Francisco Radrigán A

Ximena Raggio $\mathrm{M}$

Erna Raimann B

Jorge Rakela B

Pablo Ramírez V

Valeria Ramírez C

Simon Rascovsky

Carlos Regonesi M

Tomas Regueira $\mathrm{H}$

Paula Repetto L

Gabriela Repetto L

Pedro Retamal C

Alvaro Reyes $\mathrm{P}$

Eliana Reyes S

Enrique Reynolds $\mathrm{H}$

José Ribalta S

Attilio Rigotti R

Ernesto Ríos L

Arnoldo Riquelme P

Raúl Riquelme V

Iván Roa E

Ricardo Roa G

Juan Carlos Roa St

Juan Carlos Rodríguez D

Cristina Rodríguez S

Jorge Rodríguez T

Emilio Roessler B

Vicky Roizen G

María Graciela Rojas C

Paula Rojas G

Álvaro Rojas $G$

Christine Rojas $\mathrm{H}$

Valeria Rojas O
Alberto Rojas O

Graciela Rojas C

Ximena Rojas $\mathrm{S}$

Antonio Rollán R

Leopoldo Romero N

María Inés Romero S

Víctor Rossel M

Eduardo Rosselot J

Francisco Rothhammer E

Jaime Rozowski N

Elsa Rugiero P

Mauricio Ruiz C

Claudio Sacks P

Roque Sáenz F

Enzo Sáez H

David Sáez M

Carlos Saieh A

Sofía Salas P

Olivia Salas R

Fernando Saldías P

Hugo Sánchez R

Patricio Sandoval L

Edgar Sanhueza B

Gonzalo Sanhueza M

Emilia Sanhueza R

Julia Santín M

Manuel Santos A

Patricia Sanz C

Jorge Sapunar Z

Salvador Sarrá C

Irene Schiattino L

Ricardo Schwartz J

Manuel Sedano L

Isabel Segovia D

Erico Segovia R

Rafael Selman A

Cecilia Sepúlveda C

Rafael Sepúlveda J

Andrea Sepúlveda N

Juan Enrique Sepúlveda R

Alejandro Serani M

Leonardo Serra M

Valentina Serrano L

Carlos Silva R

Juan Silva S

Víctor Silva V

Claudio Silva Z

Teresa Sir P

Marisol Sirhan N

Patricio Smith F

Vincenza Snow 
Diana Sosa F

Juan Ramón Soto S

Rodrigo Soto $\mathrm{F}$

Marco Antonio Soza M

Freddy Squella B

Benjamín Stockins F

Valeria Stuardo A

Andrés Stutzin S

György Szánthó P

Eduardo Talesnik G

Jorge Tapia I

Renzo Tassara O

Alvaro Téllez T

Eduardo Tobar A

Vinko Tomicic F

Carlos Toro A

Rafael Torres B

Ximena Triviño B

Marcelo Trucco B

Sergio Trujillo V

Eduardo Turner G

Ricardo Uauy D

Héctor Ugalde P

Alvaro Undurraga P

María Soledad Undurraga S

Pablo Uribe G

Mario Uribe M
Sergio Uribe A

Jorge Urzúa U

Macarena Valdés C

Verónica Valdés L

Gloria Valdés St

Andrés Valdivieso D

Eduardo Valenzuela A

Jorge Valenzuela E

Raúl Valenzuela M

Sergio Valenzuela P

José Rafael Valbuena M

Carlos Vallejos V

David Vantman B

Cecilia Vargas R

Claudio Vargas R

Patricia Vásquez T

Luis Vega B

Nicolás Velasco F

Renato Verdugo L

Ismael Vergara S

Fernando Vergara E

Marcos Vergara I

Carmen Gloria Vergara L

Ivonne Vergara $\mathrm{P}$

Benjamín Vicente $\mathrm{P}$

Roque Villagra $\mathrm{C}$

Eliecer Villagra C
Eli Villalabeitia R

Marcelo Villalón C

Luis Villarroel del P

Raúl Villarroel S

Paulina Villaseca D

Miguel Villaseca $\mathrm{H}$

Jorge Villegas C

Fernando Vio del R

Joachim Vogdt

Ricardo Von Kretschmann R

Eduardo Wainstein $G$

Nelson Wohllk G

Ursula Wyneken $\mathrm{H}$

Pablo Young C

Mónica Zagolín B

Ricardo Zalaquett $S$

Jaime Zamorano G

Rodrigo Zapata L

Víctor Zárate B

Beatriz Zegers

Carlos Zehnder B

Raúl Zemelman Z

Pedro Zikto M

Sergio Zorrilla F

Ernesto Zumelzu D

Alvaro Zúñiga D 